\title{
Adult onset still's disease presenting as recurrent fever with ARDS
}

\author{
Andrey Manov*, Asha Thomas, Prabhakaran P Gopalakrishnan, Smita Subramaniam \\ JPS Health Network, 201 S Sylvania Ave, Fort Worth, TX-76111, USA
}

\begin{abstract}
Adult onset Still's disease (AOSD) is a rare inflammatory disorder characterized by daily spiking fevers, arthritis and an evanescent rash, typically affecting young adults. Nonsuppurative pharyngitis, lymphadenopathy and hepatosplenomegaly are common manifestations. We report a case of AOSD presenting as recurrent daily fevers, sore throat and lymphadenopathy. His clinical course was complicated by acute respiratory distress syndrome, which is an uncommon presentation in AOSD and has been infrequently described in literature. He responded well to steroids.
\end{abstract}

\section{Case presentation}

Our patient, a 33 year old male, presented with 1 month history of daily fever, neck pain and sore throat and a rash on his arms. During an ED visit, 3 months earlier, for right neck swelling and cough, he was diagnosed with upper respiratory tract infection and cervical lymphadenopathy. He had 10 pack year smoking history and denied alcohol or drug abuse. On exam, he had a temperature of $102.3^{\circ} \mathrm{F}$, HR 115, RR 15, BP 88/47 mm Hg and $\mathrm{SpO}_{2} 97 \%$ on room air. Neck was supple with bilateral nontender cervical lymphadenopathy. Other exam findings included nontender splenomegaly and mild maculopapular erythematous rash on chest and arms.

Lab values were significant for a WBC count of $14.6(84.8 \%$ polymorphs), hemoglobin $10.4 \mathrm{~g} / \mathrm{dl}$, hematocrit 32 , MCV 80, sodium 131, AST 93, ALT 33 and albumin 2.5. His procalcitonin was 0.6. Initial diagnosis was SIRS (Systemic inflammatory response syndrome), with likely sepsis from meningitis. Other infectious and noninfectious etiologies such as lymphoma were considered. CT scan showed enlarged cervical and mediastinal lymphadenopathy. Anemia work up showed low transferrin, normal transferrin saturation but significantly elevated ferritin of 23,350 . CSF analysis showed WBC of 21 with $95 \%$ lymphocytes, normal glucose and normal protein. ESR was elevated at 100. TB (T Spot), RPR, RF, ANA and ds-DNA were negative. Infectious workup was negative for histoplasma urine antigen, parvovirus B 19, Borrelia, Rickettsia, Bartonella, Schistosoma and CMV serologies. Bacterial, Fungal and Mycobacterial cultures from blood, urine, CSF and bone marrow were negative. Patient underwent left sided cervical lymph node excisional biopsy. Patient clinically improved and was discharged with follow up for lymph node biopsy and bone marrow biopsy results.

Patient presented two weeks later with recurrent fever, SIRS and hemoglobin of 8 . Bone marrow biopsy showed hypercellular marrow without evidence of neoplasm. Lymph Node biopsy showed follicular lymphoid hyperplasia. Presumptive diagnosis of adult onset Still's disease was entertained for Fever of Unknown Origin with workup negative for infectious and neoplastic etiologies. Patient's presentation with lymphadenopathy, arthralgia, pyrexia, sore throat, leukocytosis, elevated ferritin and splenomegaly supported the diagnosis of adult onset Still's disease.

Patient subsequently developed respiratory distress and was placed on mechanical ventilation for adult respiratory distress syndrome (ARDS). Bronchoscopy with lavage was negative for malignancy and infections. Echocardiogram showed ejection fraction of $55 \%$ and Transesophageal Echocardiogram was negative for any vegetations. He was started on intravenous methylprednisolone. He responded well with successful extubation and was later discharged on oral prednisone. Patient remained afebrile and ferritin decreased from more than 23,000 to 4,000 . Patient follows up regularly in our rheumatology clinic and is doing well. The interesting feature in our patient was ARDS which has been infrequently described in literature in Still's disease.

\section{Discussion}

Adult onset Still's disease (AOSD) is an uncommon noninfectious inflammatory disorder characterized by quotidian (daily) fevers, arthritis and an evanescent rash, typically affecting young adults from ages 16 to 35 years. Diagnosis is clinical, based on the widely used Yamaguchi criteria (Major: Fever $\geq 39^{\circ} \mathrm{C}\left(102.2^{\circ} \mathrm{F}\right)$ lasting $\geq 1$ week; Arthralgias or arthritis lasting $\geq 2$ weeks; salmon-colored skin rash during febrile episodes; Leukocytosis of $\geq 10,000 / \mu \mathrm{L}$, with $\geq 80 \%$ granulocytes. Minor: Sore throat; Lymphadenopathy; Hepatomegaly or splenomegaly; Abnormal liver function studies; Negative tests for antinuclear antibody and rheumatoid factor. Diagnosis requires minimum of five criteria with at least two of them being major criteria) [1]. Our patient had at least 3 major criteria and 3 minor criteria for AOSD.

Common laboratory findings in AOSD include neutrophilic leukocytosis, very high ESR and C-reactive protein, anemia of chronic disease, elevated ferritin levels (higher than five times the

Correspondence to: Andrey Manov, JPS Health Network, 201 S Sylvania Ave, Fort Worth, TX-76111, USA, Tel: 817-702-2478; E-mail: appenev58@aol.com

Received: August 09, 2016; Accepted: August 18, 2016; Published: August 20, 2016 
upper limits of normal) and hypoalbuminemia [1-3]. Less common AOSD manifestations include pericarditis, valvular abnormalities, thrombotic thrombocytopenic purpura, interstitial nephritis and neurological manifestations like cranial nerve palsies, seizures and aseptic meningoencephalitis. Pulmonary manifestations are infrequent and include pleurisy, cryptogenic organizing pneumonia [4], diffuse alveolar hemorrhage [5] and ARDS. Rare instances of ARDS have been described in literature in relation to AOSD [6-8] as well as treatment of AOSD with anakinra [9].

Treatment options for AOSD include nonsteroidal antiinflammatory drugs (NSAIDs), systemic corticosteroids and traditional immunosuppressants with methotrexate being the most common [3]. Anakinra [10] is the primary biological agent used in AOSD, while other agents such as infliximab, etanercept, tocilizumab and canakinumab have also been used. Alternate regimens include leflunomide, cyclosporine, intravenous immune globulin, azathioprine, cyclophosphamide, tacrolimus and gold. Periodic evaluation for disease activity and adverse effects of treatment as well as monitoring of disease markers is needed.

This case reflects the importance of considering AOSD in differential diagnosis of recurrent fever, especially in young patients and recognizing ARDS as a possible rare complication.

\section{References}

1. Yamaguchi M, Ohta A, Tsunematsu T, Kasukawa R, Mizushima Y, et al. (1992)
Preliminary criteria for classification of adult Still's disease. J Rheumatol 19: 424-430. [Crossref]

2. Cush JJ (2013) Autoinflammatory syndromes. Dermatol Clin 31: 471-480. [Crossref]

3. Kontzias A, Efthimiou P (2008) Adult-onset Still's disease: pathogenesis, clinical manifestations and therapeutic advances. Drugs 68: 319-337. [Crossref]

4. Sato H, Yokoe I, Nishio S, Onishi T, Takao T, et al. (2011) A case of adult onset Still's disease complicated with cryptogenic organizing pneumonia. Intern Med 50: 247-251. [Crossref]

5. Sari I, Birlik M, Binicier O, Akar S, Yilmaz E, et al. (2009) A case of adult-onset Still's disease complicated with diffuse alveolar hemorrhage. J Korean Med Sci 24: 155-157. [Crossref]

6. Dua AB, Manadan AM, Case JP (2013) Adult Onset Still's Disease Presenting with Acute Respiratory Distress Syndrome: Case Report and Review of the Literature. Open Rheumatol J 7: 125-128. [Crossref]

7. Guignard S, Dien G, Dougados M (2007) Severe systemic inflammatory response syndrome in a patient with adult onset Still's disease treated with the anti-IL1 drug anakinra: a case report. Clin Exp Rheumatol 25: 758-759.

8. Cheema GS, Quismorio FP Jr (1999) Pulmonary involvement in adult-onset Still's disease. Curr Opin Pulm Med 5: 305-309. [Crossref]

9. Hirohata S, Kamoshita H, Taketani T, Maeda S (1986) Adult Still's disease complicated with adult respiratory distress. Arch Intern Med 146: 2409-2410. [Crossref]

10. Kotter I, Wacker A, Koch S et al: Anakinra in patients with treatment resistant adult onset still's disease four case reports with serial cytokine measurements and a review of the literature. Semin Arthritis rheum 37: 189-197.

Copyright: ( 2016 Manov A. This is an open-access article distributed under the terms of the Creative Commons Attribution License, which permits unrestricted use, distribution, and reproduction in any medium, provided the original author and source are credited. 